\title{
Electrogram Polarity and Cavotricuspid Isthmus Block During Ablation of Typical Atrial Flutter
}

\author{
HIROSHI TADA, M.D., HAKAN ORAL, M.D., CHRISTIAN STICHERLING, M.D., \\ STEVEN P. CHOUGH, M.D., ROBERT L. BAKER, M.D., KRISTINA WASMER, M.D., \\ MICHAEL H. KIM, M.D., FRANK PELOSI, JR., M.D., GREGORY F. MICHAUD, M.D., \\ BRADLEY P. KNIGHT, M.D., S. ADAM STRICKBERGER, M.D., \\ and FRED MORADY, M.D.
}

From the Department of Internal Medicine, Division of Cardiology, University of Michigan, Ann Arbor, Michigan

\begin{abstract}
Electrogram Polarity in Atrial Flutter Ablation. Introduction: The atrial activation sequence around the tricuspid annulus has been used to assess whether complete block has been achieved across the cavotricuspid isthmus during radiofrequency ablation of typical atrial flutter. However, sometimes the atrial activation sequence does not clearly establish the presence or absence of complete block. The purpose of this study was to determine whether a change in the polarity of atrial electrograms recorded near the ablation line is an accurate indicator of complete isthmus block.

Methods and Results: Radiofrequency ablation was performed in 34 men and 10 women (age $60 \pm 13$ years [mean \pm SD]) with isthmus-dependent, counterclockwise atrial flutter. Electrograms were recorded around the tricuspid annulus using a duodecapolar halo catheter. Electrograms recorded from two distal electrode pairs (E1 and E2) positioned just anterior to the ablation line were analyzed during atrial flutter and during coronary sinus pacing, before and after ablation. Complete isthmus block was verified by the presence of widely split double electrograms along the entire ablation line. Complete bidirectional isthmus block was achieved in $39(89 \%)$ of 44 patients. Before ablation, the initial polarity of E1 and E2 was predominantly negative during atrial flutter and predominantly positive during coronary sinus pacing. During incomplete isthmus block, the electrogram polarity became reversed either only at E2, or at neither E1 nor E2. In every patient, the polarity of E1 and E2 became negative during coronary sinus pacing only after complete isthmus block was achieved. In 4 patients $(10 \%)$, the atrial activation sequence recorded with the halo catheter was consistent with complete isthmus block, but the presence of incomplete block was accurately detected by inspection of the polarity of E1 and E2.

Conclusion: Reversal of polarity in bipolar electrograms recorded just anterior to the line of isthmus block during coronary sinus pacing after ablation of atrial flutter is a simple, quick, and accurate indicator of complete isthmus block. (J Cardiovasc Electrophysiol, Vol. 12, pp. 393-399, April 2001)
\end{abstract}

atrial flutter, radiofrequency ablation, cavotricuspid isthmus

\section{Introduction}

The goal of radiofrequency ablation of typical atrial flutter is to create a line of complete block in the cavotricuspid isthmus. Conventionally, the presence of complete block has been assessed by inspection of the atrial activation sequence around the tricuspid annulus during pacing in the coronary sinus or at the inferoanterior tricuspid annulus. ${ }^{1-10}$ However, at times the atrial activation sequence may not provide a clear-cut indication of whether isthmus block is complete or incomplete. ${ }^{9,10}$

The purpose of this study was to determine whether another potential endpoint for ablation, namely, a reversal in the polarity of electrograms recorded just anterior to an intended line of block during proximal coronary sinus pac-

Supported in part by a grant from the Japanese Society of Cardiac Pacing and Electrophysiology, and by the Don Nouse Arrhythmia Research Fund.

Address for correspondence: Fred Morady, M.D., Division of Cardiology, B1F245, 1500 East Medical Center Drive, Ann Arbor, MI 48109-0022. Fax: 734-936-7026; E-mail: fmorady@ umich.edu

Manuscript received 20 October 2000; Accepted for publication 3 January 2001. ing, may be a more clear-cut indicator of complete cavotricuspid isthmus conduction block than the atrial activation sequence.

\section{Methods}

\section{Characteristics of Subjects}

The subjects of this prospective study were 44 consecutive patients who underwent radiofrequency catheter ablation of isthmus-dependent atrial flutter at the University of Michigan Medical Center. There were 34 men and 10 women (age $60 \pm 13$ years [mean $\pm \mathrm{SD}$ ]; range 33 to 79 ). Structural heart disease was present in 15 patients (34\%): coronary heart disease in 9 , nonischemic cardiomyopathy in 3 , valvular heart disease in 2 , and repaired atrial septal defect in 1 patient.

\section{Electrophysiologic Procedure}

All patients provided informed consent before the electrophysiologic procedure. Three 7- or 8-French venous sheaths were placed in a femoral vein for vascular access. A duodecapolar halo catheter (spacing between electrodes of each bipole $=2 \mathrm{~mm}$, spacing between pairs of electrodes $=$ 20-2-2-2-2-2-25-25-25 mm; Daig Corporation, Minnetonka, 


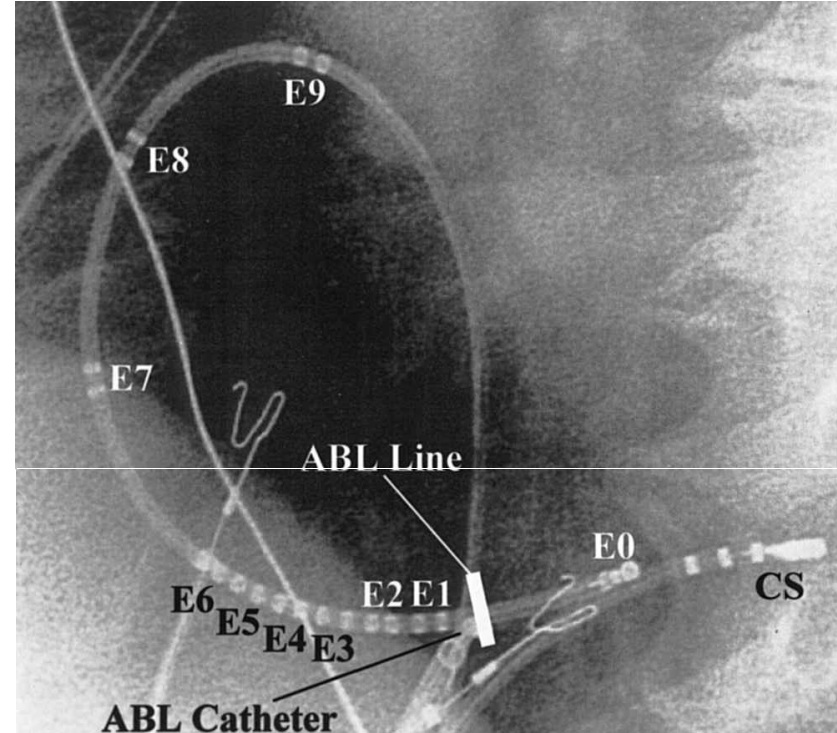

Figure 1. Left anterior oblique (45 ) fluoroscopic view of catheter positions during atrial flutter ablation. A duodecapolar halo catheter is positioned around the tricuspid annulus and along the cavotricuspid isthmus. Bipolar electrogram pairs are labeled from EO to E9. Two electrode pairs (E1 and E2) are positioned just anterior to the intended ablation line $(A B L$ Line). A quadripolar electrode catheter is positioned within the proximal coronary sinus for pacing. The ablation catheter (ABL Catheter) is positioned at the intended ablation line. The recordings displayed in Figures 2 to 5 were obtained with the catheters positioned as shown in this figure. $C S=$ coronary sinus.
MN, USA) was positioned along the tricuspid annulus, such that the distal bipole of the halo catheter was within the proximal portion of the coronary sinus (Fig. 1). Two electrode pairs separated by $2 \mathrm{~mm}$ (E1 and E2) were positioned just anterior to the intended site of ablation in the cavotricuspid isthmus (Fig. 1). A deflectable-tip, 7-French quadripolar catheter (EP Technologies, San Jose, CA, USA) was positioned within the proximal coronary sinus for pacing. A 7-French quadripolar catheter with a deflectable-tip, 4-mm distal electrode, 2-5-2 $\mathrm{mm}$ interelectrode spacing, and a thermistor embedded in the distal electrode (EP Technologies) was used to map the isthmus and deliver radiofrequency energy. A 65-cm support sheath (Daig Corporation) was used to improve catheter stability.

Bipolar electrograms were recorded with the distal electrode serving as the cathode and the proximal electrode as the anode. The bipolar electrograms were filtered at bandpass settings of 30 to $500 \mathrm{~Hz}$ and recorded digitally (EPMed Systems, Mount Arlington, NJ, USA). Electrograms were analyzed off-line at a sweep speed of 100 or $200 \mathrm{~mm} / \mathrm{sec}$. Pacing was performed from the coronary sinus or right atrium adjacent to the inferoanterior tricuspid annulus with a stimulator (model EP-3 Clinical Stimulator; EPMed Systems) at twice diastolic threshold.

Radiofrequency ablation was performed during atrial flutter in patients who presented in atrial flutter, or during pacing in the proximal coronary sinus at a cycle length of $600 \mathrm{msec}$ in patients who were in sinus rhythm. Under

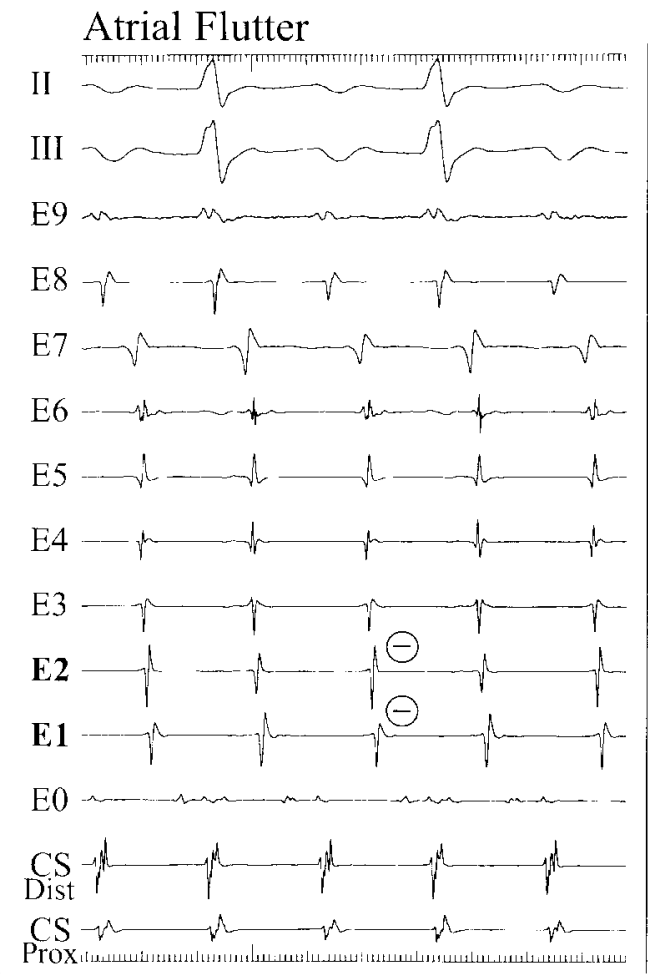

A

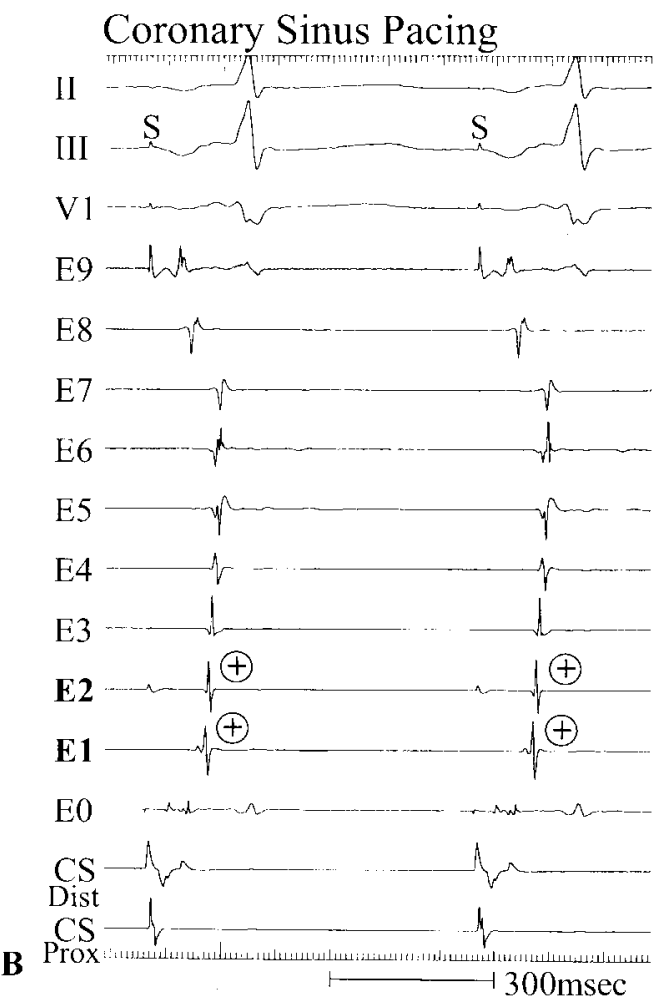

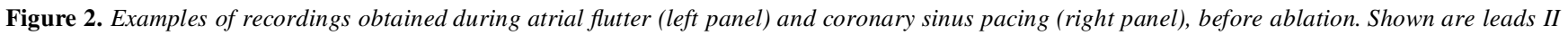

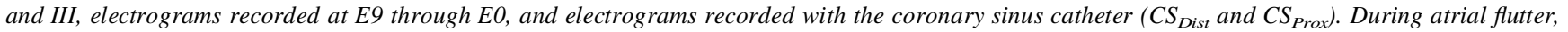

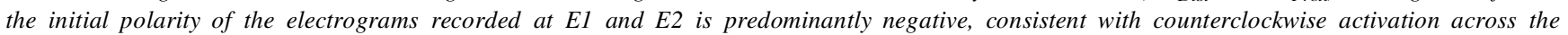

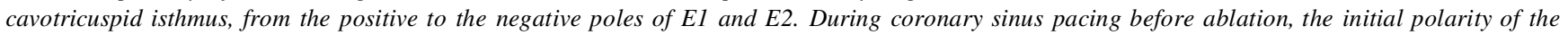

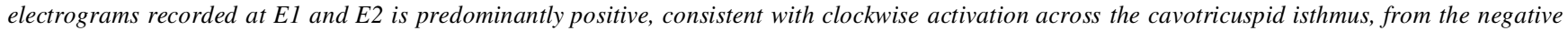
to the positive poles of E1 and E2. 
fluoroscopic guidance, individual contiguous applications of radiofrequency energy were delivered to create a line of block in the cavotricuspid isthmus inferior to the coronary sinus ostium. The power was automatically adjusted to a limit of $50 \mathrm{~W}$ to maintain a target temperature of $60^{\circ} \mathrm{C}$ at the electrode-tissue interface. Each application of energy was 45 to 60 seconds in duration.

Complete clockwise and counterclockwise isthmus block conventionally has been confirmed by analysis of the atrial activation sequence around the tricuspid annulus during pacing from the coronary sinus and inferoanterior right atrium. ${ }^{1-11}$ However, because the purpose of this study was to determine whether a change in electrogram polarity is a more accurate indicator of complete isthmus block than a change in the atrial activation sequence, the latter was not used as an endpoint for ablation. Instead, the endpoint of ablation in this study was the presence of widely split double potentials at all points along the ablation line, as described previously. ${ }^{12}$ To confirm that complete isthmus block was achieved, widely split double potentials along the entire ablation line also had to be present during an infusion of isoproterenol $2 \mu \mathrm{g} / \mathrm{min}^{13}$

\section{Results}

\section{Baseline Electrogram Polarity}

The initial polarity of E1 and E2 was predominantly negative in each of the 26 patients who were in atrial flutter upon presentation to the electrophysiology laboratory (Fig. $2 A)$. Among the 18 patients who were in sinus rhythm, the initial polarity of E1 and E2 during coronary sinus pacing always was positive (Fig. 2B).

\section{Electrogram Polarity After Complete Isthmus Block}

Complete bidirectional isthmus block, confirmed by the presence of widely split double potentials at all points along the ablation line during pacing from the coronary sinus and from the inferoanterior right atrium, was achieved in 39 (89\%) of 44 patients. In each of these 39 patients, the initial polarity of E1 and E2 during coronary sinus pacing was predominantly negative. In contrast, in the five patients in whom complete isthmus block was not achieved, the initial polarity of E1 or of both E1 and E2 always remained positive.

\section{Electrogram Polarity During Incomplete Block}

In 26 patients in this study, the transition from incomplete to complete isthmus block was documented during coronary sinus pacing. In all of these patients, the onset of complete isthmus block was associated with a transition of polarity from positive to negative at both E1 and E2. In a subset of 12 of these patients, shortly before complete isthmus block was achieved, the polarity of E2 changed from positive to negative, whereas the polarity of E1 remained positive (Fig. 3).

In seven patients, persistent complete isthmus block was preceded by a period of transient complete block. In all of these patients, the transition from complete to incomplete isthmus block was associated with a corresponding change in electrogram polarity from negative to positive at both E1 and E2 (Fig. 4).

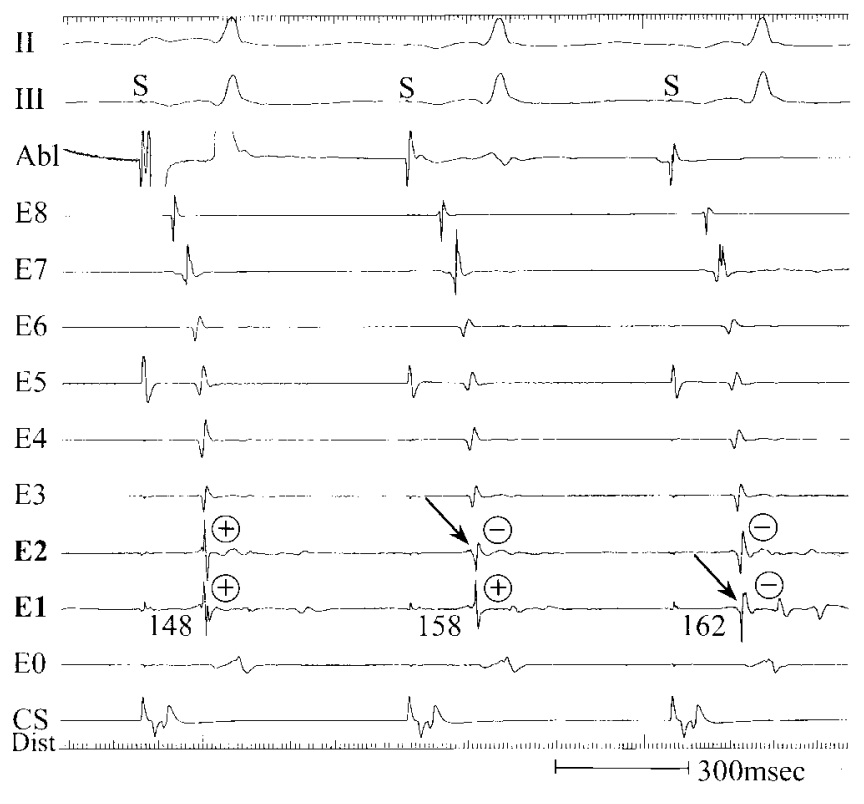

Figure 3. Example of instantaneous changes in electrogram polarity at the onset of complete cavotricuspid isthmus block. From top to bottom are leads II and III, a recording obtained with the ablation catheter (Abl) positioned on the intended ablation line in the cavotricuspid isthmus, recordings from $E 8$ to EO, and a coronary sinus electrogram $\left(C S_{\text {Dist }}\right)$. The recordings were obtained during coronary sinus pacing, in the midst of an application of radiofrequency energy. The initial electrogram polarity in the first beat is positive at both E1 and E2. Although the interval between the pacing stimulus $(S)$ and the electrogram recorded at E1 increased from a baseline value of $60 \mathrm{msec}$ (not shown) to $148 \mathrm{msec}$, the electrogram polarity at E1 and E2 indicates that there still is conduction across the ablation line. In the second beat, reversal of initial polarity only at E2 (arrow) indicates additional slowing of conduction across the ablation line (with prolongation of the stimulus-E1 interval to $158 \mathrm{msec}$ ), and collision of the clockwise and counterclockwise activation wavefronts between E1 and E2. In the third beat, the electrogram polarity becomes negative also at E1 (arrow), consistent with complete cavotricuspid isthmus block. The stimulus-E1 interval has slightly increased to $162 \mathrm{msec}$. Note that during the transition from incomplete to complete cavotricuspid isthmus block, the changes in activation sequence recorded with E8 to EO are subtle and difficult to discern. Complete cavotricuspid isthmus block was confirmed by the presence of widely split double potentials along the entire length of the ablation line.

\section{Atrial Activation Sequence During Complete and Incomplete Isthmus Block}

In each of the 39 patients in whom complete isthmus block was achieved, the atrial activation sequence around the tricuspid annulus during coronary sinus pacing and during inferoanterior right atrial pacing was consistent with complete block. Furthermore, in 22 patients in whom incomplete isthmus block was documented, the atrial activation sequence indicated the presence of some degree of conduction across the ablation line. However, in four patients in whom incomplete isthmus block was documented by the absence of widely split double potentials along the entire ablation line, the atrial activation sequence during pacing nevertheless was consistent with complete block (Fig. 5). At the time of incomplete block, the initial polarity of E1 was positive in these patients, with transition to negative polarity at the moment complete block was achieved (Fig. 5). 

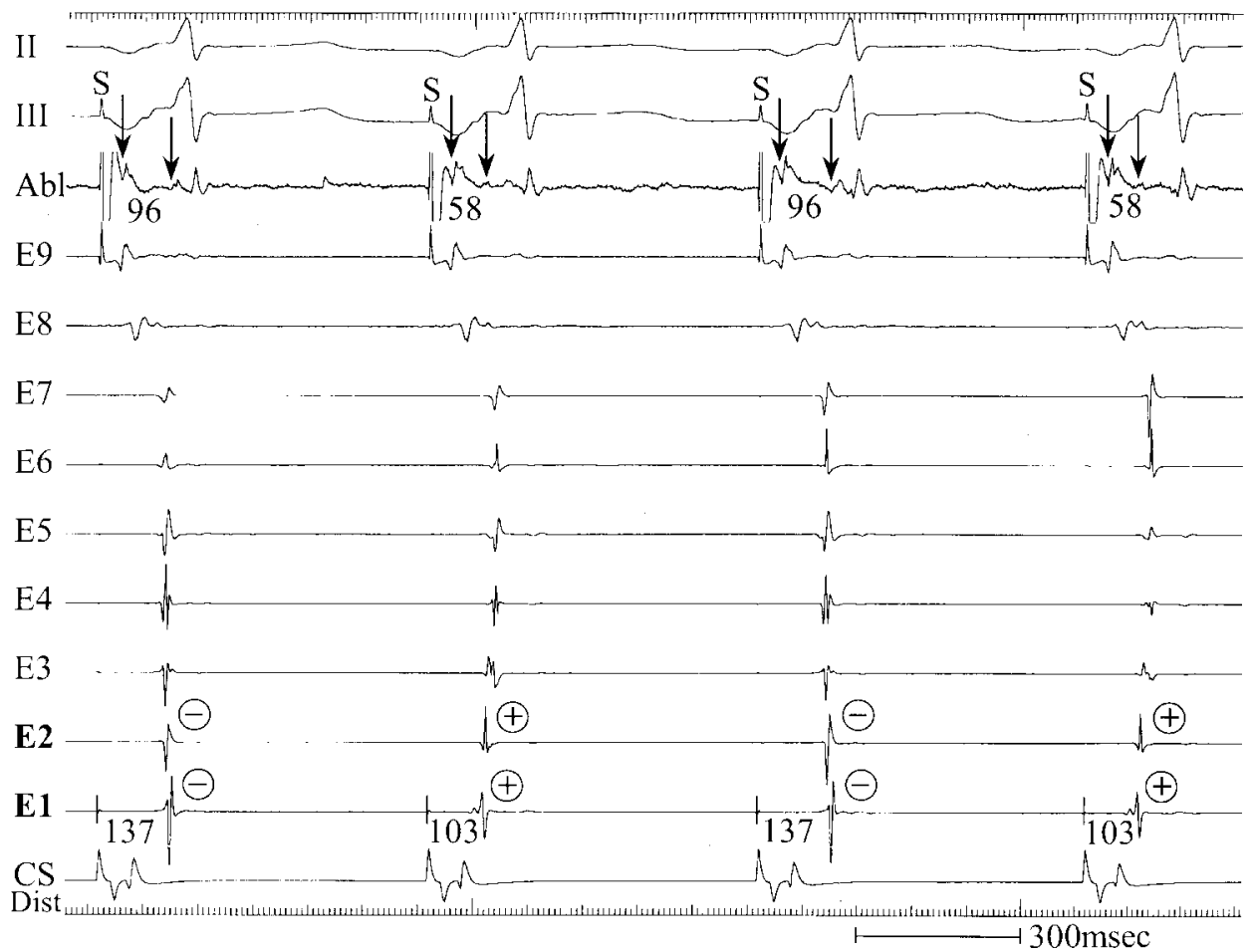

Figure 4. Example of dynamic changes in electrogram polarity during intermittent complete cavotricuspid isthmus block. From top to bottom are leads II and III, a recording obtained with the ablation catheter (Abl) positioned on the intended ablation line in the cavotricuspid isthmus, recordings from E9 to E1, and a coronary sinus electrogram $\left(C S_{D i s t}\right)$. The recordings were obtained during coronary sinus pacing, in the midst of an application of radiofrequency energy. In the first and third beats, the predominantly negative initial polarity at E1 and E2 indicates complete block, consistent with the atrial activation sequence in E9-E1, and prolongation (to $96 \mathrm{msec}$ ) of the interval between the two components of the double potential (arrows) recorded at the point of ablation. In the second and fourth beats, the initial polarity at E1 and E2 becomes positive, indicating incomplete block, consistent with the change in the atrial activation sequence around the tricuspid annulus, and with shortening to 58 msec of the interval separating the two components of the double potential recorded by the ablation catheter. The intervals designated in the E1 recording refer to the stimulus (S) to E1 interval (in milliseconds).

\section{Follow-Up}

During a mean follow-up of $11.2 \pm 4.1$ months, none of the patients in whom complete isthmus block was achieved had a recurrence of atrial flutter. Eighteen of these patients continued treatment with a Class I or III antiarrhythmic drug to prevent recurrences of atrial fibrillation. Among the five patients in whom complete isthmus block was not achieved, one had a documented recurrence of typical atrial flutter.

\section{Discussion}

\section{Main Findings}

The results of this study demonstrate that a reversal in the initial polarity of electrograms recorded just anterior to an ablation line in the cavotricuspid isthmus during proximal coronary sinus pacing is an accurate indicator of complete isthmus block. A reversal in polarity from positive to negative at recording sites just anterior to the ablation line is a more accurate indicator of complete block than is the atrial activation sequence around the tricuspid annulus. In this study, reliance on the atrial activation sequence would have resulted in an incorrect determination of complete isthmus block in $10 \%$ of patients. Incomplete isthmus block was accurately detected in these patients both by the absence of widely split double potentials along the entire ablation line and by a persistently positive electrogram polarity adjacent to the ablation line. However, whereas the presence of widely split double potentials requires sequential recordings at all points along the ablation line, electrogram polarity can be assessed instantaneously during coronary sinus pacing. Therefore, inspection of electrogram polarity adjacent to the ablation line during pacing provides a reliable and quick assessment of whether or not complete isthmus block has been achieved.

\section{Mechanism of Change in Atrial Electrogram Polarity}

During coronary sinus pacing in the baseline state, the activation wavefront progresses in a clockwise direction across the cavotricuspid isthmus, resulting in positive electrogram polarity at E1 and E2 (Fig. 6A). If there is a gap in an intended line of block in the isthmus, collision of the activation wavefronts in the clockwise and counterclockwise directions during coronary sinus pacing may occur at any point anterior to the ablation line, depending on the relative conduction velocities through the gap and around the tricuspid annulus. Therefore, when E1 and E2 are positioned just anterior to an ablation line, incomplete isthmus block is manifest as a positive electrogram polarity at both E1 and E2, or only at E1 (Figs. 6B and 6C).

Once complete isthmus block is achieved, E1 and E2 are activated only from the counterclockwise direction, result- 


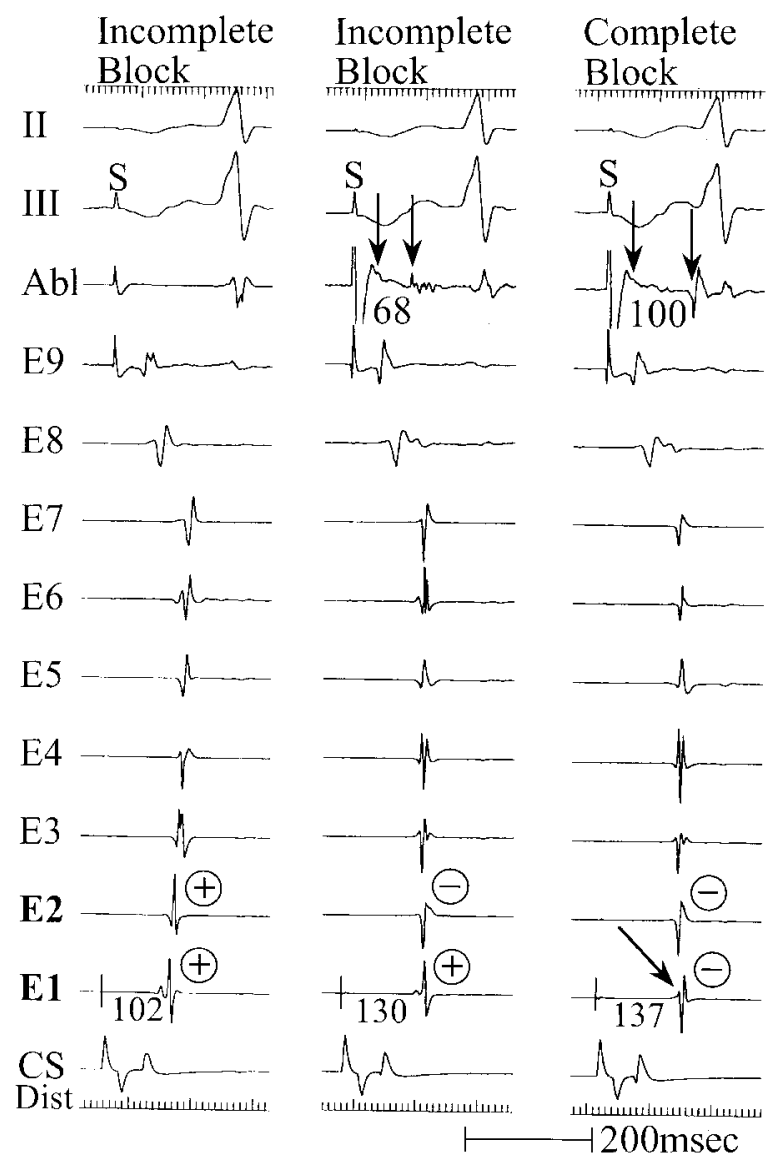

Figure 5. Example of complete cavotricuspid isthmus block that is more easily detected by changes in polarity at E1 and E2 than by changes in the atrial activation sequence around the tricuspid annulus. The format of the recordings is the same as in Figure 4. The recordings were obtained during coronary sinus pacing. After several applications of radiofrequency energy, the initial polarity at E1 and E2 is predominantly positive, indicative of persistent conduction across the cavotricuspid isthmus. This is consistent with the progression of atrial activation from E1 to E7. In the middle panel, recorded after several additional applications of radiofrequency energy in the cavotricuspid isthmus, the atrial activation sequence has changed and is consistent with complete block. However, whereas the initial polarity at E2 has become negative, the initial polarity at E1 remains positive, indicating that the block is incomplete. After one additional application of energy, the initial polarity at E1 becomes negative (oblique arrow), indicating that complete block has been achieved. This was confirmed by the presence of widely split double potentials along the entire length of the ablation line. The intervals separating the two components of the double potential recorded by the ablation catheter (vertical arrows) are indicated (in milliseconds). The intervals designated in the E1 recording refer to the stimulus (S) to E1 interval (in milliseconds).

ing in reversal of electrogram polarity from positive to negative during coronary sinus pacing (Fig. 6D).

The use of changes in electrogram polarity as an accurate indicator of complete isthmus block requires that the recording electrodes be positioned parallel to the direction of the depolarization wavefronts in the isthmus (Fig. 6). If the orientation of the electrodes is somewhat oblique to the direction of the depolarization wavefront, or if the depolarization wavefront progresses across the isthmus in a fashion that is not entirely linear, the initial polarity of an electrogram recorded in the isthmus may not be in the same direction as the predominant polarity of the electrogram. This may explain why a small initial positive deflection sometimes was present in electrograms that had a predominantly negative polarity (for example, the electrogram indicated by the oblique arrow in Fig. 5). Therefore, in this study, small initial deflections were ignored, and polarity was determined based on the predominant polarity of the first half of the electrogram.

\section{Endpoints for Ablation of Atrial Flutter}

Complete bidirectional cavotricuspid isthmus block is the endpoint for radiofrequency catheter ablation of atrial flutter. However, accurate assessment of bidirectional isthmus block has been difficult. The relatively high recurrence rate of atrial flutter in some studies probably can be attributed to the misidentification of incomplete isthmus block as complete block..$^{3,6}$

Several studies assessed transisthmus conduction by detailed atrial activation mapping using either multipolar catheters or an electroanatomic mapping system. ${ }^{14-17}$ However, very slow conduction across the isthmus may mimic complete block during activation mapping. , $^{9} 10,15$ Another study assessed the change in coronary sinus activation time during inferoanterior right atrial pacing before and after ablation as an indicator of complete isthmus block. ${ }^{18}$ It was hypothe-

A

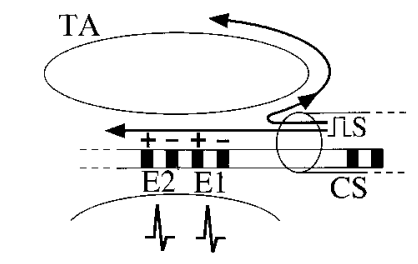

B
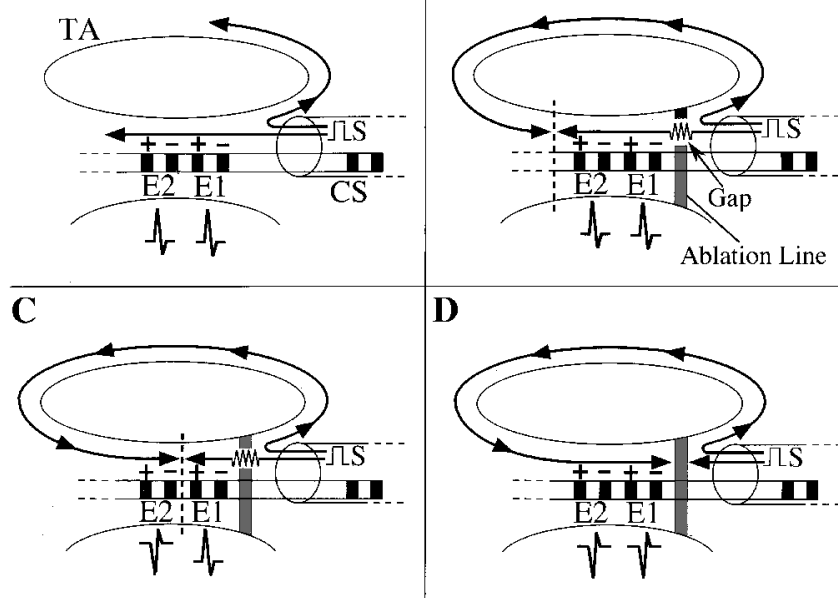

D

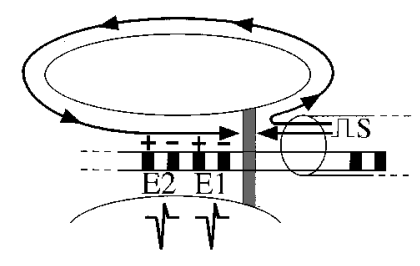

Figure 6. Schematic explanation for the changes in electrogram polarity observed during coronary sinus (CS) pacing after the creation of a line of block in the cavotricuspid isthmus. Two pairs of electrodes (E1 and E2) of a duodecapolar electrode catheter are positioned along the cavotricuspid isthmus, just anterior to the ablation line. Wavefronts resulting from pacing stimuli $(S)$ within the coronary sinus are designated by arrows exiting the CS and traveling around the tricuspid annulus (TA). (A) Before ablation, there is clockwise activation across the cavotricuspid isthmus, resulting in positive initial polarity at E1 and E2. (B) With an ablation line that contains a large gap, there is still clockwise activation across the cavotricuspid isthmus, and the initial polarity at E1 and E2 remains positive. (C) After an additional application of radiofrequency energy, the gap is still present but smaller. Conduction across the ablation line has slowed, and there is collision of clockwise and counterclockwise wavefronts in the isthmus, between E1 and E2. This results in reversal of initial polarity to negative at E2, but not at E1. (D) After a complete line of block has been created, E1 and E2 are activated only in the counterclockwise direction, resulting in an initial polarity that is negative at both recording sites. 
sized that, after complete isthmus block, the distal coronary sinus would be activated either earlier or simultaneously with the coronary sinus ostium, because of left atrial activation through Bachmann's bundle during inferoanterior right atrial pacing. This coronary sinus activation sequence was found to be $96 \%$ sensitive and $88 \%$ specific for complete isthmus block. However, the only criteria used to define complete isthmus block were the sequence of atrial activation around the tricuspid annulus and the noninducibility of atrial flutter, which both are imperfect indicators of complete block. $2,9,10,12$

The presence of double potentials on either side of a line of block was reported to indicate complete isthmus block. ${ }^{12,17}$ A continuous corridor of widely split double potentials separated by an isoelectric segment probably is the most accurate available indicator of complete isthmus block. ${ }^{12,16}$ However, detection of this endpoint requires detailed mapping of the ablation line. The use of changes in electrogram polarity as an endpoint for ablation, as described in the present study, has the major advantage of providing an instantaneous indication of whether or not complete isthmus block has been achieved.

\section{Prior Studies}

In a prior study, a reversal in local bipolar atrial electrogram polarity along the ablation line during inferoanterior atrial pacing was used to identify the site of a gap in the ablation line in patients with recurrent atrial flutter after an initial ablation procedure. ${ }^{19}$ However, the study was limited to patients with a recurrence of atrial flutter, and it was presumed that there was only one gap along the ablation line. Furthermore, the mapping catheter had to be exactly parallel to the ablation line, which may be difficult to achieve. No prior studies assessed the utility of a reversal in electrogram polarity as an indicator of complete isthmus block during catheter ablation of atrial flutter.

\section{Limitations}

A limitation of this study is that there is no clinically available indicator of complete isthmus block that is not subject to the misidentification of very slow conduction across an incomplete line of block as complete block. Therefore, in some of the patients in whom the changes in electrogram polarity were interpreted to indicate complete block, there may have been very slow conduction through a gap in the ablation line. However, there were no episodes of recurrent atrial flutter during follow-up among the patients in this study in whom it was concluded that complete isthmus block was present. Therefore, even if some of the patients in this study actually had very slow conduction across an incomplete line of block, this may not have been sufficient to allow atrial flutter to occur, and, from a functional standpoint, the identification of complete block probably was correct.

Multiple applications of radiofrequency energy may result in diminished amplitude of the atrial electrograms along the ablation line. Although this was not a problem in the present study, the reduced size of atrial electrograms may make it difficult to assess changes in polarity.

\section{Conclusion}

A reversal in electrogram polarity just anterior to an intended line of block during coronary sinus pacing after ablation of atrial flutter is a simple, accurate, and instantaneous indicator of complete isthmus block. The use of changes in electrogram polarity as an indicator of complete block is more accurate than changes in the atrial activation sequence around the tricuspid annulus. Although the presence of complete block should be confirmed by the identification of widely split double potentials along the entire ablation line, identification of changes in electrogram polarity provide a convenient indicator of isthmus block that can be assessed instantaneously after each application of radiofrequency energy.

\section{References}

1. Cosio FG, Lopez-Gil M, Goicolea A, Arribas F, Barroso JL: Radiofrequency ablation of the inferior vena cava-tricuspid valve isthmus in common atrial flutter. Am J Cardiol 1993;71:705-709.

2. Feld GK, Fleck RP, Chen PS, Boyce K, Bahnson TD, Stein JB, Calisi CM, Ibarra M: Radiofrequency catheter ablation for the treatment of human type I atrial flutter. Identification of the critical zone in the reentrant circuit by endocardial mapping techniques. Circulation 1992; 86:1233-1240.

3. Lesh MD, Van Hare GF, Epstein LM, Fitzpatrick AP, Scheinman MM, Lee RJ, Kwasman MA, Grogin HR, Griffin JC: Radiofrequency catheter ablation of atrial arrhythmias. Results and mechanisms. Circulation 1994;89:1074-1089.

4. Fischer B, Haissaguerre M, Garrigues S, Poquet F, Gencel L, Clementy J, Marcus FI: Radiofrequency catheter ablation of common atrial flutter in 80 patients. J Am Coll Cardiol 1995;25:1365-1372.

5. Blanck Z, Cetta T, Sra J, Jazayeri R, Dhala A, Deshpande S, Akhtar M: Catheter ablation of atrial flutter using radiofrequency current: Cumulative experience in 61 patients. World Med J 1998;97:43-48.

6. Calkins H, Leon AR, Deam AG, Kalbfleisch SJ, Langberg JJ, Morady F: Catheter ablation of atrial flutter using radiofrequency energy. Am J Cardiol 1994;73:353-356.

7. Nakagawa H, Lazzara R, Khastgir T, Beckman KJ, McClelland JH, Imai S, Pitha JV, Becker AE, Arruda M, Gonzalez MD, Widman LE, Rome M, Neuhauser J, Wang X, Calame JD, Goudequ MD, Jackman WM: Role of the tricuspid annulus and Eustachian valve/ridge on atrial flutter. Relevance to catheter ablation for the septal isthmus and a new technique for rapid identification of ablation success. Circulation 1996; 94:407-424.

8. Schwartzman D, Callans DJ, Gottlieb CD, Dillon SM, Movsowitz C, Marchlinski FE: Conduction block in the inferior vena caval-tricuspid valve isthmus: Association with outcome of radiofrequency ablation of type I atrial flutter. J Am Coll Cardiol 1996;28:1519-1531.

9. Poty H, Saoudi N, Nair M, Anselme F, Letac B: Radiofrequency catheter ablation of atrial flutter: Further insights into the various types of isthmus block: Application to ablation during sinus rhythm. Circulation 1996;94:3204-3213.

10. Cauchemez B, Haissaguerre M, Fischer B, Thomas O, Clementy J, Coumel P: Electrophysiological effects of catheter ablation of inferior vena cava-tricuspid annulus isthmus in common atrial flutter. Circulation 1996;93:284-294.

11. Tada H, Nogami A, Naito S, Horie Y, Suguta M, Nakatsugawa M, Hoshizaki H, Oshima S, Taniguchi K: Quantitative analysis of surface $\mathrm{P}$-wave morphology in isthmus ablation for type 1 atrial flutter: Differentiation between complete isthmus block and slow isthmus conduction. Jpn Cir J 1999;63:244-248.

12. Shah DC, Takahashi A, Jais P, Hocini M, Clementy J, Haissaguerre M: Local electrogram-based criteria of cavotricuspid isthmus block. J Cardiovasc Electrophysiol 1999;10:662-669.

13. Nabar A, Rodriguez LM, Timmermans C, Smeets JLRM, Wellens HJJ: Isoproterenol to evaluate resumption of conduction after right atrial isthmus ablation in type I atrial flutter. Circulation 1999;99: 3286-3291.

14. Johna R, Eckardt L, Fetsch T, Breithardt G, Borggrefe M: A new 
algorithm to determine complete isthmus conduction block after radiofrequency catheter ablation for typical atrial flutter. Am J Cardiol 1999;83:1666-1668.

15. Chen J, de Chillow C, Basiouny T, Sadoul N, Filho JDS, Magnin-Poull I, Messier M, Aliot E: Cavotricuspid isthmus mapping to assess bidirectional block during common atrial flutter radiofrequency ablation. Circulation 1999;100:2507-2513.

16. Shah DC, Haissaguerre M, Jais P, Fischer B, Takahashi A, Hocini M, Clementy J: Simplified electrophysiologically-directed catheter ablation of recurrent common atrial flutter. Circulation 1997;96:25052508 .
17. Shah DC, Haissaguerre M, Jais P, Takahashi A, Hocini M, Clementy $\mathrm{J}$ : High-density mapping of activation through an incomplete isthmus ablation line. Circulation 1999;99:211-215.

18. Tsai CF, Chen SA, Tai CT, Chiang CE, Yu WC, Chen YJ, Feng AN, Hsieh MH, Ding YA, Chang MS: Impact of transisthmus ablation of typical atrial flutter on coronary sinus activation time. PACE 2000;23: 63-73.

19. Yamabe H, Okumura K, Misumi I, Fukushima H, Ueno K, Kimura Y, Hokamura Y: Role of bipolar electrogram polarity mapping in localizing recurrent conduction in the isthmus early and late after ablation of atrial flutter. J Am Coll Cardiol 1999;33:39-45.

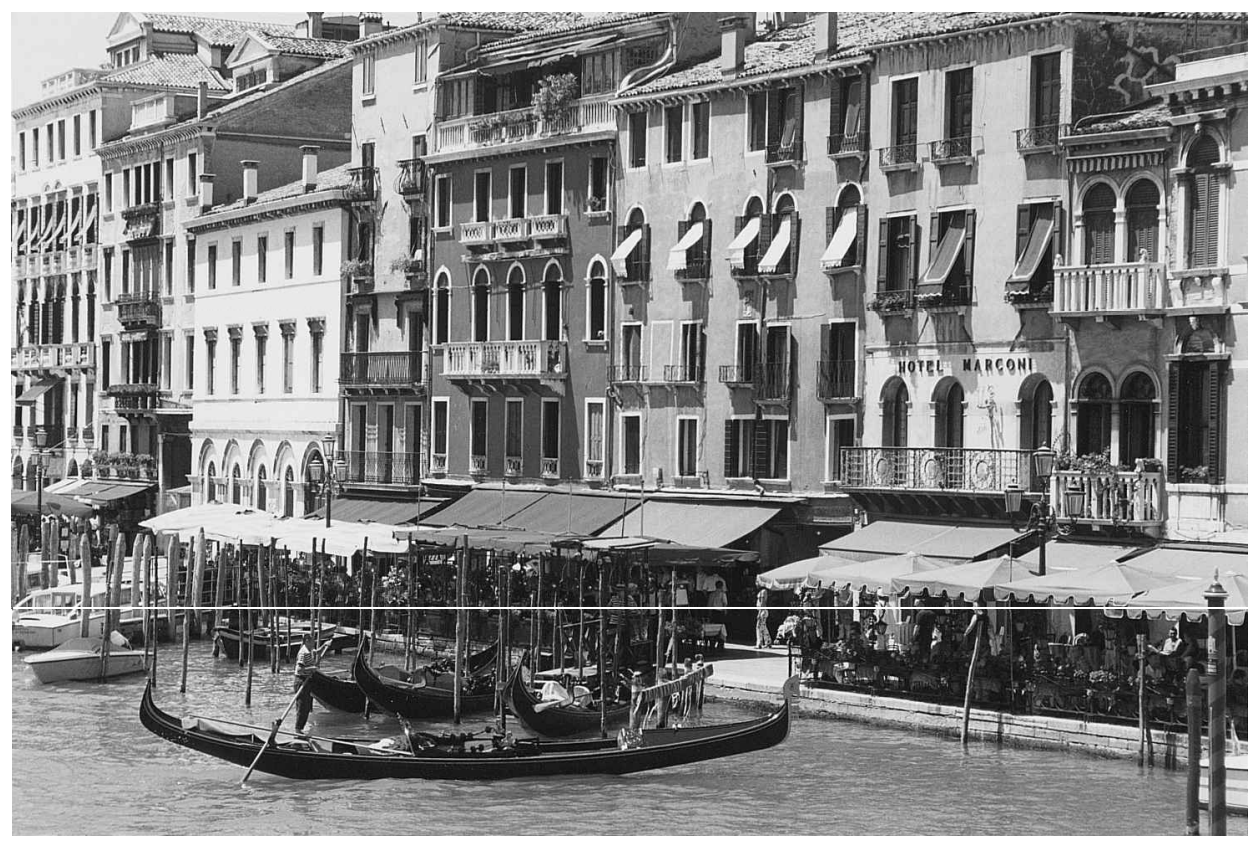

Venice, Italy 